\title{
Do we need cost-effectiveness analysis of brachytherapy for cervical cancer in the 3D image-guided era?
}

\author{
Suzumura EA ${ }^{1 *}$, Gama LM² de Carvalho $\mathrm{HA}^{2}$ and de Soárez PC ${ }^{1}$ \\ ${ }^{1}$ Departamento de Medicina Preventiva, Faculdade de Medicina FMUSP, Universidade de Sao Paulo, Sao Paulo, SP, Brazil \\ ${ }^{2}$ Departamento de Radiologia e Oncologia, Divisao de Radioterapia, Faculdade de Medicina FMUSP, Universidade de Sao Paulo, Sao Paulo, SP, Brazil
}

\begin{abstract}
Cervical cancer is the second most prevalent cancer in the female population. Intracavitary brachytherapy is considered an essential component of the disease management. In conventional brachytherapy, treatment planning is performed using X-ray imaging to visualize the pelvic bones structures and applicators, considering point $\mathrm{A}$ as the reference for dose delivery. For the last years, three-dimensional (3D) image-guided brachytherapy, with the use of computed tomography or magnetic resonance imaging, has been used to determine tumor volume and shape, as well as the healthy organs at risk, and prescribed dose is delivered to an 'at-risk' volume. Although 3D brachytherapy shows technical advantage and potentially superior clinical outcomes over conventional, 3D planning does carry added costs. The decision about incorporating new health technologies in health care systems should be based in multiple aspects, including financial issues. Economic evaluation studies have been adopted as a method for supporting efficient resource allocation. Cost-effectiveness analysis, in particular assessing consequences as quality-adjusted life years (QALYs) or disability-adjusted life years (DALYs), that is cost-utility analysis, are the preferred type of economic evaluation study for decision-making on technology incorporation. Evidence from economic evaluation studies are not transferrable to other health care systems with distinct health policies, resources and priorities, therefore, whether the benefits afforded by $3 \mathrm{D}$ brachytherapy are cost-effective is to be determined withing each health care system considering local context.
\end{abstract}

\section{Introduction}

Cervical cancer is the second most prevalent cancer in the female population [1]. In 2018, the World Health Organization (WHO) estimated that 570,000 new cases were diagnosed, and approximately 311,000 women died of cervical cancer in the world, with $85 \%$ of them in middle- and low-income countries [1].

The American Cancer Society estimates that in 2020 about 13,800 new cases of invasive cervical cancer will be diagnosed and more than 4,200 women will die from cervical cancer in the United States [2]. Cervical cancer was once one of the most common causes of death in American women but, as a result of the increase in human papillomavirus (HPV) screening, death rates have dropped. Nonetheless, cervical cancer mortality rates have not changed much over the last 10 years [2].

In Brazil, the National Cancer Institute (INCA) estimates that 16,590 new cases will be diagnosed in 2020 , with an estimated risk of 15.43 cases per 100,000 women [3]. In 2017, more than 6,300 women died from cervical cancer in Brazil [3].

\section{Treatment of cervical cancer}

Radiotherapy is an excellent cervical cancer treatment modality because of the tolerance of the cervix to high radiation doses [4]. The conventional radiotherapy regimen involving conformal external beam radiotherapy of the pelvis followed by intracavitary brachytherapy, and concomitant chemoradiation, has been the standard of care for locally advanced tumors since the early 90's [4].

Derived from ancient Greek words for 'short distance' (brachios) and 'treatment' (therapy), brachytherapy refers to therapeutic use of encapsulated radionuclides within or close to a tumor. It is sometimes called Curietherapy, named after its French inventor Pierre Curie [5]. Since 1900 brachytherapy has been used in the treatment of cancer and is considered an essential component of the disease management [6].

Unlike external beam conformal radiotherapy, in which the tumor receives radiation from a source located outside the body, brachytherapy involves a more precise placement of radiation sources close to tumor site. The greater effectiveness of brachytherapy compared to external radiotherapy is attributed to the ability to provide high concentrated and more accurate doses to the target tissue, promoting greater local control and increasing the safety of adjacent healthy tissues [7]. In contrast, brachytherapy is invasive, and requires the insertion of specific applicators usually under sedation or anesthesia [5].

In conventional gynecological brachytherapy, treatment planning is performed using two-dimensional (2D) X-ray imaging to visualize the pelvic bones structures and applicators, using anatomic references for planning, without conforming to the tumor shape and size [4]. Treatment planning relies on dose points, considering point $\mathrm{A}$ as the reference for dose delivery. Tumor volume coverage is the most important goal for local control. The accurate determination of the gross tumor volume (GTV), the clinical target volume (CTV) involving the tumor volume and safety margins, as well as the healthy organs

${ }^{*}$ Correspondence to: Erica Aranha Suzumura, Departamento de Medicina Preventiva, Faculdade de Medicina FMUSP, Universidade de Sao Paulo. Av Dr Arnaldo 455, CEP: 01246903, Sao Paulo, SP, Brazil, E-mail: esuzumura@yahoo.com.br

Key words: brachytherapy, uterine cervical neoplasms, economic evaluations, costeffectiveness analysis

Received: March 13, 2020; Accepted: March 25, 2020 Published: March 30, 2020 
at risk (OAR) surrounding the tumor are crucial for better clinical outcomes [4]. In addition, the dose offered decreases rapidly as the distance from the applicator increases, emphasizing the importance of proper placement of the applicators guided by some imaging exam [6].

Grade 3-4 hematological toxicity, enteritis and cystitis are often the most limiting adverse events associated to conventional chemoradiotherapy for cervical cancer and can compromise the effectiveness of treatment due to unscheduled interruptions, which allows tumor regrowth and influence directly on patients quality of life [4]. Therefore, whenever possible it is important to choose a radiotherapy technique that protects OAR from excessive toxicity in order to reduce the acute side effects and late complications of radiotherapy and potentially improve local control through improved target coverage [4].

\section{D image-guided brachytherapy}

For the last years, three-dimensional (3D) image-guided brachytherapy, with the use of computed tomography (CT) or magnetic resonance imaging (MRI), has been used in clinical practice [8]. This was a move away from prescribing dose to point A to an 'at-risk' volume (predominantly, the high-risk CTV [HR-CTV]) [9]. The evaluation of dose to OAR has also shifted away from the ICRU 38 reference points to a dose volume histogram (DVH) based approach, allowing brachytherapy plans to more accurately define where dose will be, rather than predicting where it may be [10]. The main advantage of $3 \mathrm{D}$ brachytherapy is the quality of the $3 \mathrm{D}$ image and the opportunity for dose optimization to more accurately conform to the CTV. In small tumors, the irradiated volume can be reduced to decrease the dose to $\mathrm{OAR}$, and in larger tumors, the isodose prescription can be expanded for better coverage $[6,9]$.

\section{Clinical effects of 3D compared to 2D brachytherapy}

Few systematic reviews were published in this field. None of them attempted to assess clinical outcomes associated with $3 \mathrm{D}$ compared to $2 \mathrm{D}$ brachytherapy in patients with cervical cancer. They aimed to report the clinical effects of only one of the image-planning strategies [11-13], or to compare the technical usefulness and dosimetry of $3 \mathrm{D}$ and $2 \mathrm{D}$ imaging technologies for brachytherapy planning, without reporting clinical outcomes [14].

Randomized trials are the gold standard to assess the effects of treatments, however such trials were not published so far. Indeed, these would provide the best evidence to compare both techniques. However, as in many other radiation treatments, $2 \mathrm{D}$ techniques may be associated with a higher level of imprecision on dose delivery and toxicity. This is not different regarding brachytherapy, that is supposed to deliver very high doses to the target. A comprehensive evaluation of tumor volume (GTV, CTV and HR-CTV) and OAR is provided by 3D brachytherapy, which is being increasingly used worldwide. This can be considered as a reflection of the same phenomenon that occurred with external beam conformal radiotherapy, resulting in a gradual transition from $2 \mathrm{D}$ to 3D technique.

For instance, the emerging evidence from the large multicentre international EMBRACE studies points to a relationship of higher dose delivery and more advanced techniques with better outcomes $[15,16]$. In the RetroEMBRACE study, improved local control was associated with an overall survival benefit of about $10 \%$ compared with historical cohorts, with limited severe toxicity [15].
Retrospective observational studies comparing 3D and 2D brachytherapy showed increased local control [17-19], reduced toxicity rates $[17,18,20]$, which resulted in improved overall survival $[17,21]$ in patients treated with 3D approach. A French multicentric nonrandomized prospective study assessing the clinical effects of the two planning strategies showed that $3 \mathrm{D}$ brachytherapy also improved local control with half the toxicity observed with 2D dosimetry [22-24].

Cervical cancer and its treatment had a negative influence on the quality of life of these women. Some studies showed negative effects associated to brachytherapy on the domains related to symptoms and functionality, which impacts overall quality of life [25]. In this sense, as $3 \mathrm{D}$ brachytherapy is associated with lower toxicity and late adverse effects rates $[17,18,20,22-24]$, it has the potential to reduce symptoms and the time to functional recovery, and to improve sexual function after the treatment, enhancing patients quality of life.

$3 \mathrm{D}$ brachytherapy is still evolving, and until now, the results are promising. Improving in local control, survival rates and quality of life, are really expected by radiation oncologists in the near future.

\section{Understanding economic evaluation studies}

The assessment of the effectiveness and safety of an intervention are some of the components of the decision-making process regarding policies for incorporating new health technologies in health care systems. Financial resources are finite and the use of resources in a new technology usually determines reallocation from another area [26]. In this sense, not only the expected health benefits, but also the costs are important issues in the decision.

Economic evaluation studies have been adopted as a method for supporting efficient resource allocation. Data on the effectiveness of an intervention are more easily "transferable" from one geographic region to another, however, the generalization of results of health economic studies is limited, requiring the production of reliable information contextualized for each country population [26].

For a better understanding, two types of economic evaluation studies can be identified in the literature: full and partial studies. Full economic evaluation are defined as studies in which (1) two or more alternative interventions are compared, and (2) both costs and effects (consequences or benefits or outcomes) of the compared treatments are taken into account [27]. Full economic evaluation studies are regarded as the optimal type of economic evaluation because they are specifically designed to inform policy decisions on technology incorporation in a health care system. In a partial economic evaluation study, the two noted requirements (comparison two treatments and measurement of both costs and effects) are not met, and they usually report only costs as outcomes [27].

Four types of full economic evaluation studies can be distinguished: cost-effectiveness analysis, cost-utility analysis, cost-benefit analysis, and cost-minimization analysis. In a cost-effectiveness analysis, costs are expressed in monetary values (e.g. Euros, Dollars, Reais, etc.) and effects in natural units, such as reduction in toxicities, increase in local control or in survival rates [27]. Cost-utility analysis is a special type of cost-effectiveness analysis, where the costs are also valued in monetary values and effects are typically measured as quality-adjusted life years (QALYs) or disability-adjusted life years (DALYs) [27].

In cost-benefit analysis, the benefits of health care are expressed to an equivalent amount of consumption, that is, the amount of money that an individual is willing to pay or to receive in return for the benefits 
offered. In this type of economic evaluation, both costs and effects are expressed in monetary terms of the alternative interventions [27].

For cost-minimization analysis the effectiveness of the compared interventions is equivalent, thus the main element of the analysis is the determination of which of the alternatives will entail lower costs [26]. Although cost-minimization analysis is distinguished as a full economic evaluation study [26], it is considered as only a costing exercise and not a formal economic evaluation for some decision makers, therefore, it is not an appropriate reference case analysis [28].

Economic evaluation studies can be based on a modelling study, in which various secondary sources of data (e.g. literature, population databases) might be used to build a model simulating the course of the disease and costs associated, assuming that patients were submitted to each of the alternative treatments. Also, economic evaluation studies can be trial-based, in which the data for economic analyses are collected alongside data from a clinical trial [27]. A third approach is also possible using a combination of the previous analytical approaches.

Cost-effectiveness analysis, in particular assessing consequences as QALYs or DALYs, that is cost-utility analysis, are the preferred type of economic evaluation study for both decision-making on technology incorporation in a health care system and clinical practice guidelines development.

The result of cost-effectiveness analysis can be expressed in a summary measure called incremental cost-effectiveness ratio (ICER), with is defined as the difference in expected costs between two alternative interventions divided by the difference in expected effects $[27,28]$. It represents the average incremental cost associated with one additional unit of the measure of consequences (e.g., cases avoided, increase in survival, QALYs or DALYs). The ICER can be estimated as presented below, where $C_{i}$ and $E_{i}$ are the cost and effect in patients who received (or would receive) the intervention; and $C_{a}$ and $E_{a}$ are the cost and effect in patients who received (or would receive) the alternative usual treatment:

\section{ICER $=\left(C_{i}-C_{a}\right) /\left(E_{i}-E_{a}\right)$}

An intervention with a lower ICER (e.g., spending US\$ 1000 on an intervention that would increase QALYs by 1 year) would indicate a good value for money intervention, while a higher ICER (e.g., spending US $\$ 1,000,000$ to increase QALYs by 1 year) would typically be considered a low value for money intervention [29]. Nevertheless, objectively, what defines if an intervention is cost-effective is known as "willingness-to-pay threshold".

A willingness-to-pay threshold represents an estimate of what a consumer of health care might be prepared to pay for the health benefit given other competing demands on that consumer's resources [30]. Therefore, to set a willingness-to-pay threshold many relevant factors, such as the opportunity cost and affordability within the context of the health care system, have to be taken in account.

The use of any single willingness-to-pay threshold is ultimately arbitrary and is widely criticized in health policy circles [29]. Researchers have attempted in various ways to deduce what constitutes a reasonable threshold on the basis of economic theory or empirical estimates [31], however an international debate is currently under way on the need for countries to adopt explicit thresholds. For instance, the United States decision makers use variable thresholds, instead of rigidly fixed ones, as rough guides to help determine whether particular investments constitute reasonable value [32].
There are critics arguing that willingness-to-pay thresholds are unable to consider all the important social preferences and values, equity and distributive fairness [33]. Health technology assessment researchers agree that additional studies and efforts are needed to develop thresholds that clearly incorporate budget constraints and opportunity costs $[33,34]$. Therefore, WHO emphasizes that a fixed willingness-to-pay threshold should never be used as a stand-alone criterion for decision-making and its estimation is a challenge for health care systems [30].

\section{Cost-effectiveness analysis of $3 \mathrm{D}$ compared to $2 \mathrm{D}$ brachytherapy}

Although 3D brachytherapy shows technical advantage and potentially superior clinical outcomes over conventional technique, 3D planning does carry added costs. Typically, 3D brachytherapy requires the acquisition of cross-sectional imaging for treatment planning with each fraction of brachytherapy [6,9]. This adds not only additional time for image acquisition and simulation, but it requires available imaging machines, that generally are used not only for brachytherapy, and increases length of treatment planning time and their associated costs.

Whether the benefits afforded by 3D brachytherapy are costeffective is to be determined withing each health care system considering local context. For instance, Kim et al. investigated the cost-effectiveness of 3D brachytherapy compared to conventional $2 \mathrm{D}$ brachytherapy for the treatment of locally advanced cervical cancer from the perspective of Medicare US system [35]. They used a modelbased approach, where identical hypothetical cohorts treated with five fractions of intracavitary brachytherapy after external radiation therapy were followed during three years. For 3D brachytherapy, treatment was performed as either CT-based or MRI-based plan. The authors showed that the 3D CT-based strategy costs US\$ 3003 more than 2D brachytherapy while gaining 0.16 QALYs, resulting in an ICER of US\$ 18,634 per QALY gained. The 3D MRI-based strategy costs US\$ 4476 more than 2D brachytherapy, resulting in an ICER of US\$27,774 per QALY gained [35]. As the willingness-to-pay threshold adopted was US\$ 50,000/QALY gained, the 3D strategies were considered costeffective technologies compared with 2D brachytherapy [35].

As discussed in the previous section, the results of the study published by Kim et al. are not transferrable to other health care contexts with distinct health policies, insurance and reimbursement systems, resources and priorities.

Health care systems that progressively transitioned from $2 \mathrm{D}$ conventional to 3D brachytherapy, making their decision not-based on cost-effectiveness analyses, may consider such evaluations futile. Full economic evaluation studies become more relevant with the increasing cost scrutiny in health care, particularly in middle- and low-income countries as Brazil.

\section{Conclusion}

Technologies to treat cancer have received the most attention in the recent years, not only because of the increase of cases, but also because of the costs involved. Facing the promising, but still not definitive superiority of $3 \mathrm{D}$ compared to $2 \mathrm{D}$ brachytherapy, our research group is conducting a systematic review with meta-analyses to compare the effects of the two image planning technologies on clinical outcomes in patients with cervical cancer. A prospective cohort study will assess quality of life in patients treated with the two planning strategies. A systematic review of economic evaluation studies will be also carried 
out to inform parameters to support the choice and construction of a model to perform a cost-effectiveness analysis of the treatment of cervical cancer with 3D brachytherapy versus $2 \mathrm{D}$ from the perspective of the Brazilian Unified Health System (Sistema Único de Saúde, SUS). These results may support the decision-making process about the incorporation of $3 \mathrm{D}$ brachytherapy in cancer programs, the development of clinical guidelines and national health policies.

\section{Authorship and contributorship}

Suzumura drafted the original manuscript. All authors made substantive intellectual contributions. All authors revised and approved the final manuscript.

\section{Funding information}

This study was partially supported by the Coordenação de Aperfeiçoamento de Pessoal de Nível Superior - Brasil (CAPES) Finance Code 001. De Soarez PC is the recipient of grant from the Conselho Nacional de Desenvolvimento Cientifico e Tecnológico (CNPq, National Council of Technological and Scientific Development; Research Grant No. 302268/2019-7).

\section{Competing interest}

The authors have no conflict of interests to declare.

\section{References}

1. Human papillomavirus (HPV) and cervical cancer (2018) WHO.

2. Key statistics for cervical cancer (2020) Am Cancer Soc.

3. Brasil (2018) Ministério da Saúde. Incidência de Câncer no Brasil. Estimativas. Inst Nac Câncer.

4. Dutta S, Nguyen NP, Vock J, Kerr C, Godinez J, et al. (2015) Image-Guided Radiotherapy and -Brachytherapy for cervical cancer. Front Oncol 5: 1-6.

5. Skowronek J (2017) Current status of brachytherapy in cancer treatment - short overview. J Contemp Brachytherapy 9: 581-589. [Crossref]

6. Haie-Meder C, Pötter R, Van Limbergen E, Briot E, De Brabandere M, et al. (2005) Recommendations from Gynaecological (GYN) GEC-ESTRO Working Group (I): Concepts and terms in 3D image based 3D treatment planning in cervix cancer brachytherapy with emphasis on MRI assessment of GTV and CTV. Radiother Oncol 74: 235-45.

7. Han K, Milosevic M, Fyles A, Pintilie M, Viswanathan AN (2013) Trends in the utilization of brachytherapy in cervical cancer in the United States. Int J Radiat Oncol Biol Phys 87: 111-119.

8. Pötter R, Tanderup K, Kirisits C, de Leeuw A, Kirchheiner K, et al. (2018) The EMBRACE II study: The outcome and prospect of two decades of evolution within the GEC-ESTRO GYN working group and the EMBRACE studies. Clin Transl Radiat Oncol 9: 48-60.

9. Potter R, Haie-Meder C, Van Limbergen E, Barillot I, De Brabandere M, et al. (2006) Recommendations from gynaecological (GYN) GEC ESTRO working group (II): concepts and terms in 3D image-based treatment planning in cervix cancer brachytherapy-3D dose volume parameters and aspects of 3D image-based anatomy, radiation physics, radiobiolog. Radiother Oncol 78: 67-77.

10. Georg P, Lang S, Dimopoulos JCA, Dörr W, Sturdza AE, et al. (2011) Dose-volume histogram parameters and late side effects in magnetic resonance image-guided adaptive cervical cancer brachytherapy. Int J Radiat Oncol Biol Phys 79: 356-362. [Crossref]

11. Wang F, Tang Q, Lv G, Zhao F, Jiang X, et al. (2017) Comparison of computed tomography and magnetic resonance imaging in cervical cancer brachytherapy: A systematic review. Brachytherapy 16: 353-365.

12. Salem A, Salem AF, Al-Ibraheem A, Lataifeh I (2011) Evidence for the use PET for radiation therapy planning in patients with cervical cancer: a systematic review. Hematol Oncol Stem Cell Ther 4: 173-181.

13. Mendez LC, Weiss Y, D’Souza D, Ravi A (2017) Three-dimensional-guided perinealbased interstitial brachytherapy in cervical cancer: A systematic review of technique, local control and toxicities. Radiother Oncol 123: 312-318.
14. D'Souza D, Baldassarre F, Morton G, Falkson C, Batchelar D (2011) Imaging technologies for high dose rate brachytherapy for cervical cancer: A systematic review. Clin Oncol 23: 460-475.

15. Sturdza A, Pötter R, Fokdal LU, Haie-Meder C, Tan LT, et al. (2016) Image guided brachytherapy in locally advanced cervical cancer: Improved pelvic control and survival in RetroEMBRACE, a multicenter cohort study. Radiother Oncol 120: 428-433.

16. Fokdal L, Sturdza A, Mazeron R, Haie-Meder C, Tan LT (2016) Image guided adaptive brachytherapy with combined intracavitary and interstitial technique improves the therapeutic ratio in locally advanced cervical cancer: Analysis from the retroEMBRACE study. Radiother Oncol 120: 434-440.

17. Kang HC, Shin KH, Park SY, Kim JY (2010) 3D CT-based high-dose-rate brachytherapy for cervical cancer: Clinical impact on late rectal bleeding and local control. Radiother Oncol 97: 507-513.

18. Rijkmans E, Nout R, Rutten I, Ketelaars M, Neelis K, et al. (2014) Improved survival of patients with cervical cancer treated with image-guided brachytherapy compared with conventional brachytherapy. Gynecol Oncol 135: 231-238.

19. Ribeiro I, Janssen H, De Brabandere M, Nulens A, De Bal D (2016) Long term experience with $3 \mathrm{D}$ image guided brachytherapy and clinical outcome in cervical cancer patients. Radiother Oncol 120: 447-454.

20. Derks K, Steenhuijsen JLG, Berg HA Van Den, Houterman S, Cnossen J (2018) Impact of brachytherapy technique ( $2 \mathrm{D}$ versus $3 \mathrm{D}$ ) on outcome following radiotherapy of cervical cancer. J Contemp Brachytherapy 10: 17-25.

21. Lindegaard JC, Fokdal LU, Nielsen SK, Juul-Christensen J, Tanderup K (2013) MRI-guided adaptive radiotherapy in locally advanced cervical cancer from a Nordic perspective. Acta Oncol 52: 1510-1519.

22. Charra-Brunaud C, Harter V, Delannes M, Haie-Meder C, Quetin P (2012) Impact of 3D image-based PDR brachytherapy on outcome of patients treated for cervix carcinoma in France: results of the French STIC prospective study. Radiother Oncol 103: 305-313.

23. Métayer Y, Meyer P, Brunaud C, Peiffert D (2009) Quality control in pulsed dose rate brachytherapy. Cancer/Radiotherapie 13: 318-22.

24. Charra-Brunaud C, Peiffert D (2008) Preliminary results of a French prospectivemulticentric study of 3D pulsed dose-rate brachytherapy for cervix carcinoma. Cancer/ Radiotherapie 12: 527-531.

25. Sabulei C, Maree JE (2019) An exploration into the quality of life of women treated for cervical cancer. Curationis 42: 1-9.

26. Brasil (2014) Secretaria de ciência tecnolgia e insumos estratégicos. departamento de ciência e tecnologia. ministério da saúde. Diretrizes Metodológicas: Diretriz de Avaliação Econômica.

27. Drummond M, Sculpher M, Claxton K, Stoddart G, Torrance G (2015) Methods for the economic evaluation of health care programmes. 4th Ed. Oxford: Oxford University Press.

28. CADTH Methods and Guidelines (2017) Guidelines for the economic evaluation of health technologies. 4th Ed. Canada.

29. Vu CC, Jawad MS, Krauss DJ (2020) The cost-effectiveness and value proposition of brachytherapy. Semin Radiat Oncol 30: 87-93.

30. Bertram MY, Lauer JA, De Joncheere K, Edejer T, Hutubessy R (2016) Costeffectiveness thresholds: Pros and cons. Bull World Health Organ 94: 925-930.

31. Neumann PJ, Cohen JT, Weinstein MC (2014) Updating cost-effectiveness - The curious resilience of the \$50,000-per-QALY threshold. N Engl J Med 371: 796-797. [Crossref]

32. Grosse S (2008) Assessing cost-effectiveness in healthcare: history of the $\$ 50,000$ per QALY threshold. Expert Rev Pharmacoecon Outcomes Res 8: 165-178.

33. Soarez PC De, Novaes HMD (2017) Cost-effectiveness thresholds and the Brazilian Unified National Health System. Cad Saude Publica 33: e00040717.

34. Pitt C, Vassall A, Teerawattananon Y, Griffiths U, Guinness L (2016) Foreword: Health economic evaluations in low- and middle-income countries: methodological issues and challenges for priority setting. Heal Econ 25: 1-5.

35. Kim H, Rajagopalan MS, Beriwal S, Huq MS, Smith KJ (2015) Cost-effectiveness analysis of $3 \mathrm{D}$ image-guided brachytherapy compared with $2 \mathrm{D}$ brachytherapy in the treatment of locally advanced cervical cancer. Brachytherapy 14: 29-36. [Crossref]

Copyright: (C2020 Suzumura EA. This is an open-access article distributed under the terms of the Creative Commons Attribution License, which permits unrestricted use, distribution, and reproduction in any medium, provided the original author and source are credited. 